Aim of the study: Different carcinomas have different characteristics, which may play a crucial role in diagnosis and treatment. Our study was aimed at understanding the development pattern of bone metastasis from hepatocellular carcinoma, based on its imaging characteristics, so as to provide a more targeted treatment.

Material and methods: Forty two patients (123 lesions) with hepatocellular carcinoma hospitalized from June 2006 to June 2011 underwent radiotherapy for bone metastasis in our department. Clinical and imaging data were analyzed retrospectively (based on CT imaging, also with reference to $\mathrm{MRI}$, ECT, or PET$\mathrm{CT}$, etc.).

Results: One hundred of 123 lesions were vertebral metastases; 23 were non-vertebral. The major form of bone destruction was osteolytic change. Metastasis in the vertebral body was found in $87.8 \%$, and lesions were well distributed in various sections. Vertebral appendix metastasis accounted for $52 \%$, where lesions could be independent of vertebral body metastasis. Formation of a soft tissue mass in bone metastasis was found in $68.6 \%$ of all patients. The center of the mass from a vertebral body metastasis was mostly located at the site of the lesion; masses from the vertebral appendix and the pelvis, on the other hand, often presented as a "peripheral mass". Masses were not formed in lesions with pure osteoblastic changes. Conclusions: The most common radiographic feature is an osteolytic lesion, either replaced by soft tissue mass, or invaded by soft tissue mass from the vicinity, which often cause compression syndrome. Vertebral appendix metastasis can exist independently from vertebral body metastasis, which should be paid more attention to avoid missed diagnosis.

Key words: hepatocellular carcinoma, bone metastasis, vertebrae, spinal cord compression, computer assisted radiographic image interpretation.

\section{Radiographic characteristics of bone metastases from hepatocellular carcinoma}

\author{
Hai-yan Chen, Xiu-mei Ma, Yong-rui Bai
}

Department of Radiation Oncology, Renji Hospital, afflicated to School of Medicine, Shanghai Jiao Tong University Shanghai, China

Hepatocellular carcinoma (HCC) is one of the most common carcinomas. Approximately 626,000 new patients are diagnosed with HCC globally each year; among them $50 \%$ are in China, with a fatality rate of $94 \%$; that is, approximately 600000 deaths each year [1]. Due to development of diagnosis and treatment in recent years, the overall survival rate of HCC patients has increased. However, there is also a higher chance for bone metastasis; prognosis of bone metastasis from HCC is still poor, regardless of active treatment [2-4]. Current knowledge of radiographic characteristics of bone metastasis from HCC is still limited, and detailed manifestation in one specific lesion was rarely involved. Analysis of the characteristics of bone lesions helps to better understand what they are before understanding why they exist, and provides support for more targeted treatment. In this study, we studied retrospectively the clinical and radiographic characteristics of 42 patients (123 lesions) with bone metastasis from HCC.

\section{Material and methods}

\section{Patients}

A total of 42 HCC patients hospitalized from June 2006 to June 2011 were treated for bone metastasis with radiotherapy (Table 1); 33 were male, and 9 were female. The average age was 53 years (ranging from 17 to 75 years). Among these patients, 20 had Karnofsky performance status (KPS) > 80; 17 were 50-80; 5 were $<80$. These 5 patients were mainly hospitalized with limb movement disorders due to lesions from bone metastasis and required health care by their family; among them 4 patients were paraplegic.

Among 42 patients, 29 presented with pathologic diagnosis of HCC; the other 13 patients without pathologic diagnosis, nonetheless, met the Diagnostic Standard of Primary Liver Cancer (Chinese Anticancer Association) [5, 6]. Thirteen of them had a history of hepatitis B infection. 36 patients underwent treatment for HCC before they were diagnosed with bone metastases. The average interval between the diagnosis of HCC and bone metastasis was 10 months (ranging from 2 to 60 months). Among them 26 underwent surgical treatment: 16 orthotopic liver transplantation (OLT), 10 hepatic segmentectomy. 14 underwent transcatheter arterial chemoembolization (TACE), 5 radiofrequency ablation (RFA), 2 systemic chemotherapy, and 3 were administered sorafenib. In addition, 6 patients had bone metastasis from HCC as the first manifestation, 2 of whom underwent TACE after diagnosis.

Among these 42 patients, 12 had pulmonary metastases, 6 had abdominal lymph node metastases, 3 had brain metastases, 2 had adrenal metastases and 1 had renal metastases. The primary lesions were not under control in 28 patients. 


\section{Diagnosis of bone metastasis}

All 42 patients suffered from bone metastasis related symptoms: 38 patients had different levels of pain; 11 experienced neurological symptoms such as paresthesia, myasthenia or incontinence. All patients had undergone at least one of the following tests: bone scintigraphy using 99mTc-MDP (99Tm-ECT), computed tomography (CT), magnetic resonance imaging (MRI), PET-CT using 18F-FDG (18F-FDG PET-CT). All were diagnosed as having bone metastasis. A total of 26 patients underwent MRI (61.9\%), Twerrty-three ECT (54.5\%), 11 CT scans (26.2\%) and 3 PET-CT scans (7.1\%). 23 patients underwent two types of tests or more; and all 11 patients with CT scans underwent at least another test.

\section{Imaging data access and analysis}

A CT scan was performed with patients' consent, using a GE HiSpeed CT scanner. The patient was put in a supine position, and a vacuum pad or thermoplastic mask was used for fixation. The range of scanning for vertebral metastasis should include at least 2 upper and lower vertebrae; for metastasis in limbs, the proximal joint of the bone should be included; for costal and sternum metastasis, the entire thoracic cavity should be included; and for pelvic metastasis, the entire pelvis should be included. Slice thickness was $5 \mathrm{~mm}$; and for certain vertebrae, $3 \mathrm{~mm}$ if necessary. A plain scan was applied under normal conditions. An enhanced scan was applied only when visceral condition was reviewed as well.

Images were delivered to the Unix system, and were analyzed independently by two radiologists, both with at least 5 years' work experience, using GE's in-built image analyzer. Internal lesion distribution on the cross section was primarily observed, and expansion of the lesion was determined by the $3 \mathrm{D}$ reconstruction image, with reference to medical history, MRI, PET-CT, and ECT results available. Consultation with a third doctor (with 15 years' work experience) was arranged when there was a disagreement. Observation of bone mass destruction was conducted under the bone window (window level: 400; window width: 2000), while observation of soft tissue mass was conducted under the soft tissue window (window level: 60; window width: 360).

Metastatic lesions were classified as follows: 1) osteolytic destruction presented on CT scans as destruction or even loss of trabeculae in spongy bones; the margin could be well or poorly defined; 2) osteoblastic change presented as increased density of spot or flake-like shadow; the margin was generally well defined; 3) mixed change presented as co-existence of osteoclastic and osteoblastic change. For patients with vertebral metastasis, imaging characteristics of nonmetastatic vertebrae should also be considered.

To describe the distribution of lesions on the cross section objectively, we referred to the Weinstein-Boriani-Biagini (WBB) surgical staging system [7], and divided each vertebra into 12 radial zones, as shown in Fig. 1 . Sectors 1-3: the right appendix sector; sectors 4-6: the right vertebral body sector; sectors 7-9: the left vertebral body sector; sectors 10-12: the left appendix sector. The zone of lesion distribution and its type (osteoblastic, osteoclastic, mixed) were recorded; spinal canal stenosis or formation of soft tissue was also recorded.
Table 1. General state of health of 42 patients with bone metastasis from $\mathrm{HCC}$

\begin{tabular}{|c|c|c|}
\hline Sex & $\begin{array}{l}\text { male } \\
\text { female }\end{array}$ & $\begin{array}{r}33 \\
9\end{array}$ \\
\hline Age & average 53 (range: 17-75) & \\
\hline KPS & $\begin{array}{l}>80 \\
50-80 \\
<50\end{array}$ & $\begin{array}{r}20 \\
17 \\
5\end{array}$ \\
\hline $\begin{array}{l}\text { Hepatitis B } \\
\text { infection }\end{array}$ & $\begin{array}{l}\text { yes } \\
\text { no }\end{array}$ & $\begin{array}{l}30 \\
12\end{array}$ \\
\hline $\begin{array}{l}\text { Pathological } \\
\text { diagnosis }\end{array}$ & $\begin{array}{l}\text { yes } \\
\text { no }\end{array}$ & $\begin{array}{l}29 \\
13\end{array}$ \\
\hline $\begin{array}{l}\text { History of } \mathrm{HCC} \\
\text { treatment }\end{array}$ & $\begin{array}{l}\text { yes } \\
\text { no }\end{array}$ & $\begin{array}{r}39 \\
4\end{array}$ \\
\hline Therapy & $\begin{array}{l}\text { liver transplantation } \\
\text { hepatic segmentectomy } \\
\text { TACE } \\
\text { radio frequency } \\
\text { full-body chemotherapy } \\
\text { administration of sorafenib }\end{array}$ & $\begin{array}{r}16 \\
10 \\
16 \\
5 \\
2 \\
3\end{array}$ \\
\hline $\begin{array}{l}\text { Liver lesion } \\
\text { control }\end{array}$ & $\begin{array}{l}\text { controlled } \\
\text { uncontrolled }\end{array}$ & $\begin{array}{l}14 \\
28\end{array}$ \\
\hline $\begin{array}{l}\text { Metastases } \\
\text { in other } \\
\text { organs }\end{array}$ & $\begin{array}{l}\text { lung } \\
\text { abdominal lymph nodes } \\
\text { brain } \\
\text { adrenal gland } \\
\text { kidney }\end{array}$ & $\begin{array}{r}12 \\
6 \\
3 \\
2 \\
1\end{array}$ \\
\hline $\begin{array}{l}\text { Discovery of bone } \\
\text { metastasis }\end{array}$ & $\begin{array}{l}\text { during treatment } \\
\text { In first visit }\end{array}$ & $\begin{array}{r}36 \\
6\end{array}$ \\
\hline
\end{tabular}

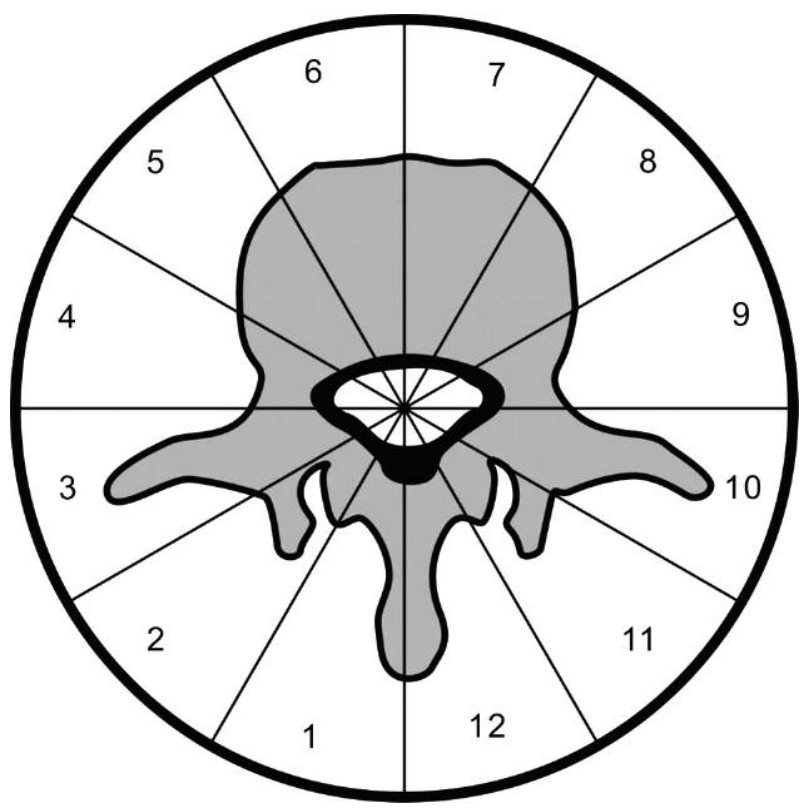

Fig. 1. WBB staging system 


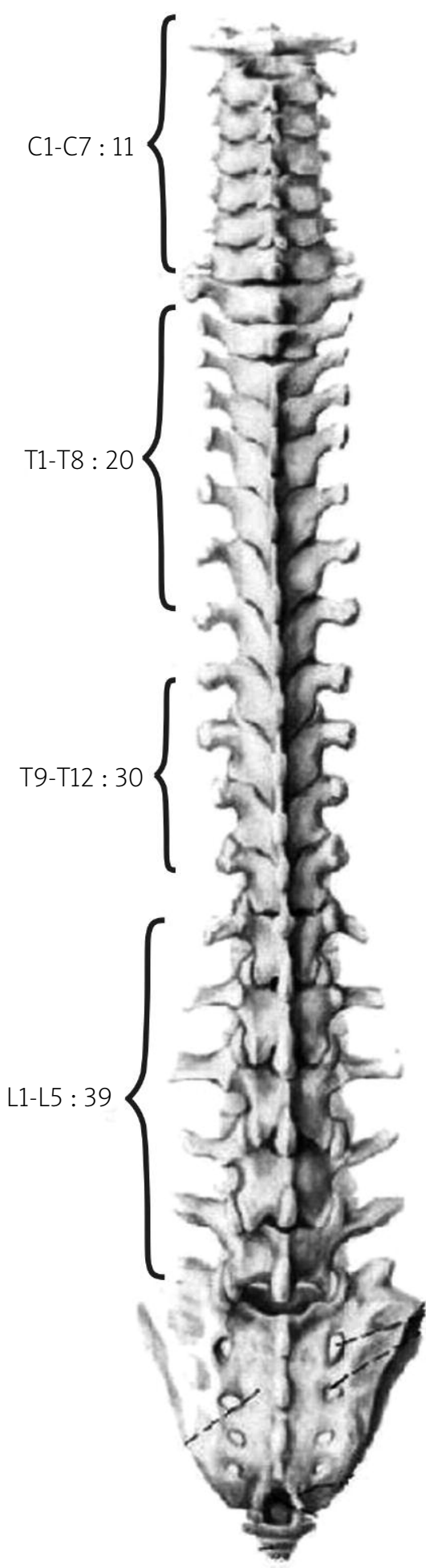

Fig. 2. Distribution of metastatic spinal lesions

\section{Results}

General conditions of bone metastasis

Among 42 patients, 24 had only vertebral metastasis, 8 had non-vertebral bone metastasis; the other 10 had both vertebral and non-vertebral metastasis. A total of 100 vertebral metastases and 23 non-vertebral metastases were recorded. One patient showed abnormal radionuclide accumulation in $\mathrm{C} 7$ and $\mathrm{T} 1$ on bone scans; other factors like trauma were ruled out. The patient was diagnosed with bone metastasis with consideration of clinical data and bone scan results; however, CT scan showed no visible bone destruction. Thus, a total of 98 vertebrae with visible lesions were analyzed.

Among 121 analyzable lesions, 81.8\% were osteolytic (99 of 121); $7.4 \%$ were osteoblastic (9 of 121); $10.7 \%$ were mixed (13 of 121). Soft tissue mass formation was found in $68.6 \%$ of them (83 of 121 ).

\section{Distribution and manifestation of vertebral lesions}

Distribution of lesions

Involvement of the lower thoracic and lumbar spine accounted for the majority in 100 vertebral metastases. The top three were: 10 in L2, 9 in T12 and T9 (Fig. 2).

\section{Distribution of lesions based on the WBB staging system}

In 98 vertebrae with visible metastatic lesions, a total of 1176 sectors were counted (the WBB staging system divides each vertebra into 12 radial zones). 446 of 1176 sectors were observed with lesions. Involvement of sectors 4-9 (i.e. the vertebral body sector) accounted for $74.4 \%$ (332/446), and no significant difference in the distribution in each sector was observed. Involvement of sectors 1-3 and sectors 10-12 (i.e. the appendix sector) accounted for $25.6 \%$ (114/446); the lowest proportion of lesions appeared in sector 12 and 1 (Table 2).

\section{Involvement of the vertebral body and appendix}

In these 98 vertebral metastases, a total of 86 (87.8\%) metastases of the vertebral were observed, 47 of which were simple metastases of the vertebral body. Fifty-one (52.0\%) appendix metastases were observed, among which 12 existed alone. The other 39 were appendix metastasis combined with metastasis of the vertebral body (as shown in Fig. 3); for these 39 cases, lesions would merge into flakes, or exist separately as well.

\section{Type of lesion}

In our group of study, 76 showed osteolytic changes; lesions presented as flake-like or erosion-like decreases in bone density, with a poorly defined margin. Nine showed osteoblastic changes, which presented as a patchy increase in bone density, with a well-defined margin. Thirteen showed mixed changes, which generally presented as osteolytic bone

Table 2. Distribution of metastatic lesions in different vertebral sectors

\begin{tabular}{|c|c|c|c|c|c|c|c|c|c|c|c|c|}
\hline Sector & 1 & 2 & 3 & 4 & 5 & 6 & 7 & 8 & 9 & 10 & 11 & 12 \\
\hline Count & 10 & 17 & 23 & 59 & 58 & 57 & 53 & 55 & 50 & 30 & 24 & 10 \\
\hline
\end{tabular}



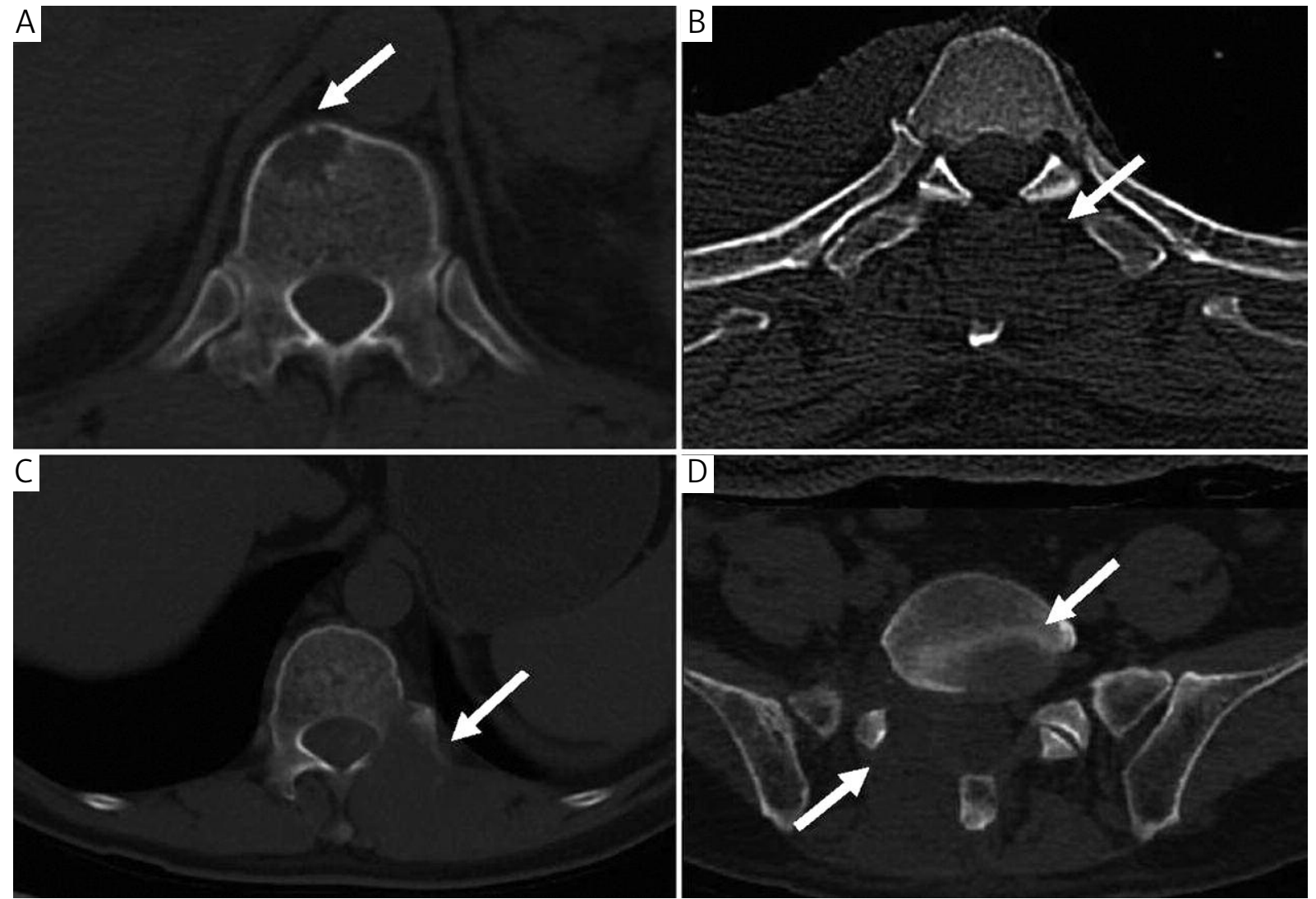

Fig. 3. A - 52-year-old man, CT showed single vertebral metastasis in the right vertebral sector of T12 (indicated by arrow; B - 39-yearold man, CT showed single bone destruction in the appendix sector of T2, with no penetration into the vertebral body, soft tissue mass encysted the spinous process (indicated by arrow); C - The same patient CT showed bone destruction in the left appendix sector of T11, where soft tissue mass extended from the appendix sector to the posterior edge of the left vertebra (indicated by arrow); D - Patient with separate metastases in vertebral body and appendix in L5 (indicated by arrow); soft tissue mass formed at the posterior edge of the left vertebra, a separate metastasis observed at the posterior edge of the left vertebral body
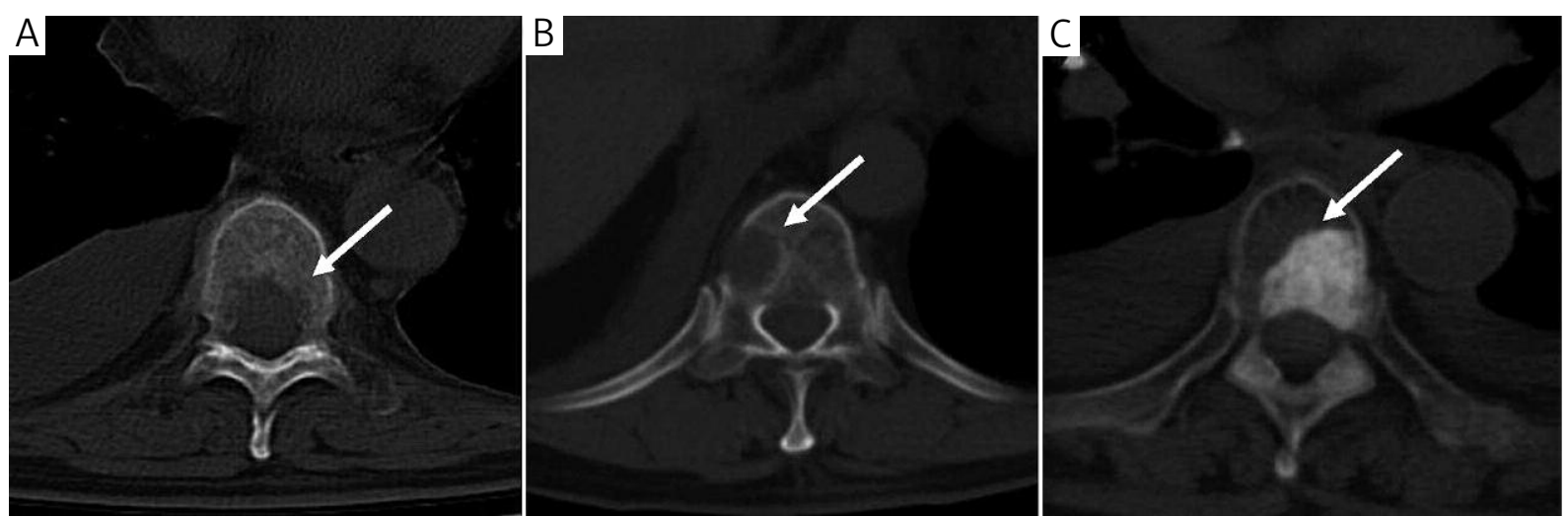

Fig. 4. A - 43-year-old man, CT (bone window) showed bone destruction with pure osteolytic change in T9 (indicated by arrow); B - 52-yearold man, CT showed multiple bone destruction with mixed change in T11; an osteoblastic ring was visible around the osteolytic bone destruction site, with discontinuous cortex (indicated by arrow); C - 46-year-old man, CT showed bone destruction with pure osteoblastic change in T6 (indicated by arrow)

mass destruction with reactive osteogenic ring formation in the periphery (Fig. 4).

\section{Formation of soft tissue mass}

Formation of soft tissue mass was observed in 60 of 98 vertebral metastases; 35 of them presented as normal bone matrix replaced by soft tissue mass, which showed expansive growth; some mass broke through the cortex and grew in the vicinity. This kind of growth could often be seen in the vertebral body. The other 25 soft tissue masses presented as paravertebral masses, which grew inwards to encyst and destroy the bone matrix. The center of the mass seemed not to be located at the metastatic lesion; hence it was named 
a "peripheral mass". The mass usually failed to be distinguished from muscles around the vertebra, and even an MRI scan would not be able to determine if it was a muscular lesion invading the bone mass, or a cancer embolism planted on the periosteum and thereby forming a lesion which grew inwards, causing bone destruction, and outwards, forming a mass (which often appeared in the appendix sector) (Figs. 5 and 6). Formation of a metastatic mass in the appendix sector accounted for 38 of 51 (74.5\%). Twenty one CT scans with clear spinal canal stenosis were observed, which accounted for $21.4 \%$ (21 of 98).

\section{Distribution and type of non-vertebral metastases}

In 23 non-vertebral metastatic lesions, 13 pelvic, 3 costal, 2 sternal, 2 cranial (occipital and temporal, respectively), 2 femoral and 1 scapular metastases were observed. All 23 lesions were osteolytic changes, which presented as replacement by soft tissue mass. Twenty of them broke through the cortex and grew in the periosteal gap (Figs. 5 and 6); the other 3 presented as expansive growth.

\section{Discussion}

Due to the improved duration of control of the primary tumor site as well as improved imaging, bone metastases from HCC are noted much more frequently. Bone metastases induce pain, vertebral instability, vertebral compression, limb movement disorders and so forth. Quality of life for patients is severely compromised; some eventually died because of the lesion. Due to different biological behaviors and growing features of different types of tumor, it is necessary to have in-depth knowledge of features of each tumor, in order to provide a more targeted treatment. Current knowledge of CT characteristics of bone metastasis from HCC is still lim- ited. We reviewed the imaging characteristics of 42 patients (123 lesions) with bone metastatic lesions.

In our study, most lesions were located at the vertebrae; the pelvis took second place, followed by the ribs, sternum, limb bones and cranium, which showed no significant difference from other types of tumors [8]. Destruction of bone matrix mostly presented as osteolytic change, which was also similar to the results of previous retrospective studies [9]. Our data showed that in vertebral metastasis, the incidence of lower-thoracic and lumbar metastasis was high. Apart from the fact of a rich blood supply in thoracic and lumbar vertebrae, some characteristics of HCC itself might also be relevant. Most HCC patients had a history of liver disease. In our study, the morbidity of hepatitis B was $71.4 \%$; these patients then developed cirrhosis, and vast collateral circulation was formed between the portal vein system, abdominal and pelvic vein system. Cancer thromboses may travel through the collateral circulation to the vertebrae, and plant at the surface of the vertebrae and the medullary cavity through an anastomotic vascular network. Collateral circulation in HCC patients was primarily located in lower thoracic and lumbar vertebrae; thus metastases in these sections were more common.

In previous studies on vertebral metastasis, detailed distribution in one specific location was rarely involved (apart from a few case reports); data on bone metastasis from HCC were even sparser. Due to restrictions of imaging methods, a handful of existing studies tended to focus more on the general distribution and macroscopic characteristics of bone lesions, and detailed information was lacking [10, 11]. Our study took advantage of CT imaging as well as MRI, ECT, PET-CT and so forth; thus detailed distribution and characteristics of lesions in different locations were analyzed.

To objectively describe the distribution of vertebral lesions, we made use of the WBB staging system [7], in the
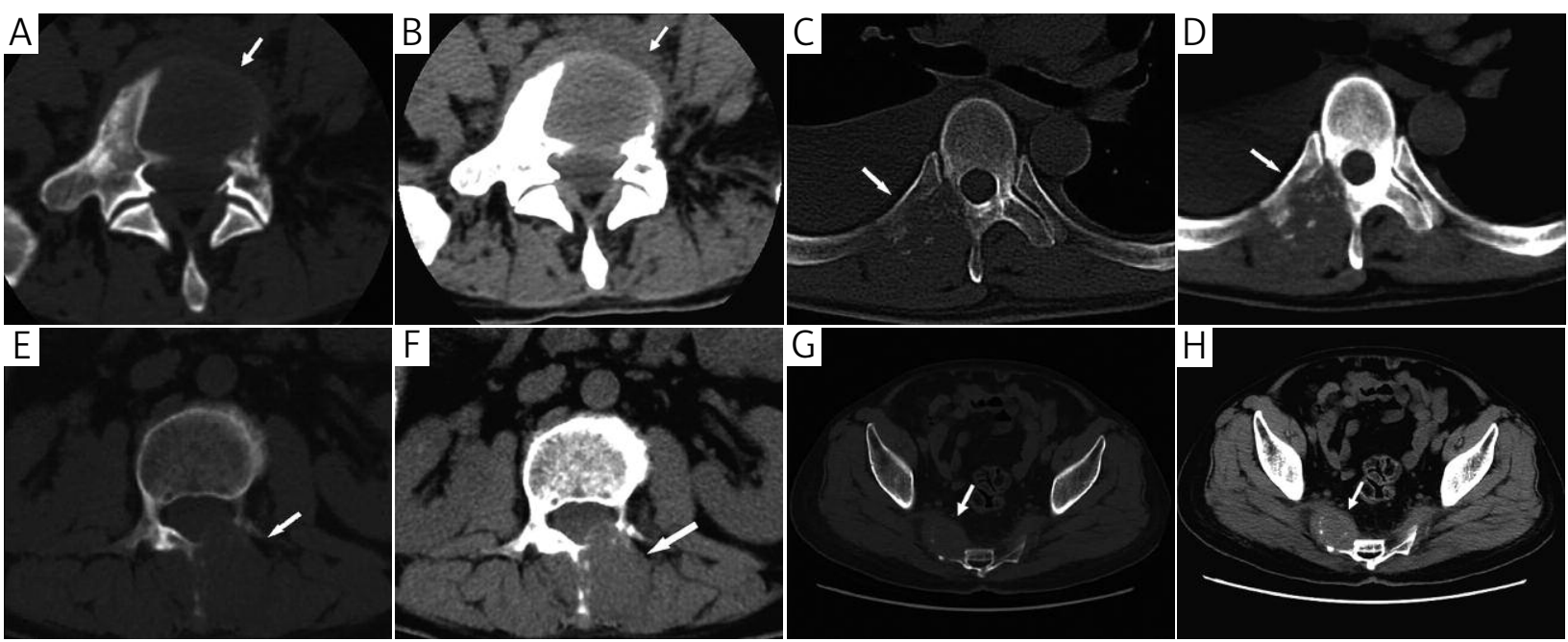

Fig. 5. A, B - 65-year-old man, CT (bone window and soft tissue window) showed bone destruction in L4, and was replaced by soft tissue mass, which penetrated through the cortex with paravertebral growth (indicated by arrow); C, D - 41-year-old man, CT showed peripheral soft tissue mass in the right appendix sector in T5, encysting the right transverse process and pedicle of vertebral arch, adjacent ribs were also involved (indicated by arrow); E, F-39-year-old man, CT showed peripheral soft tissue mass in the left appendix sector in L3; mass was poorly defined; it invaded the left vertebral lamina and spinous process, and also penetrated through the vertebral lamina into the vertebral canal; narrowed spinal canal was observed (indicated by arrow); G, H - Patient observed with a well-defined soft tissue mass in the right sacrum (indicated by arrow) 
hope of specifying a predilection site of the lesion. As a result, the vertebral body was found to be a major location for metastatic lesions, which accounted for $74.4 \%$; distribution was relatively even between sectors, and no predilection site was observed; $25.6 \%$ of lesions were observed in the appendix sector, among which sectors 1 and 12 were the least
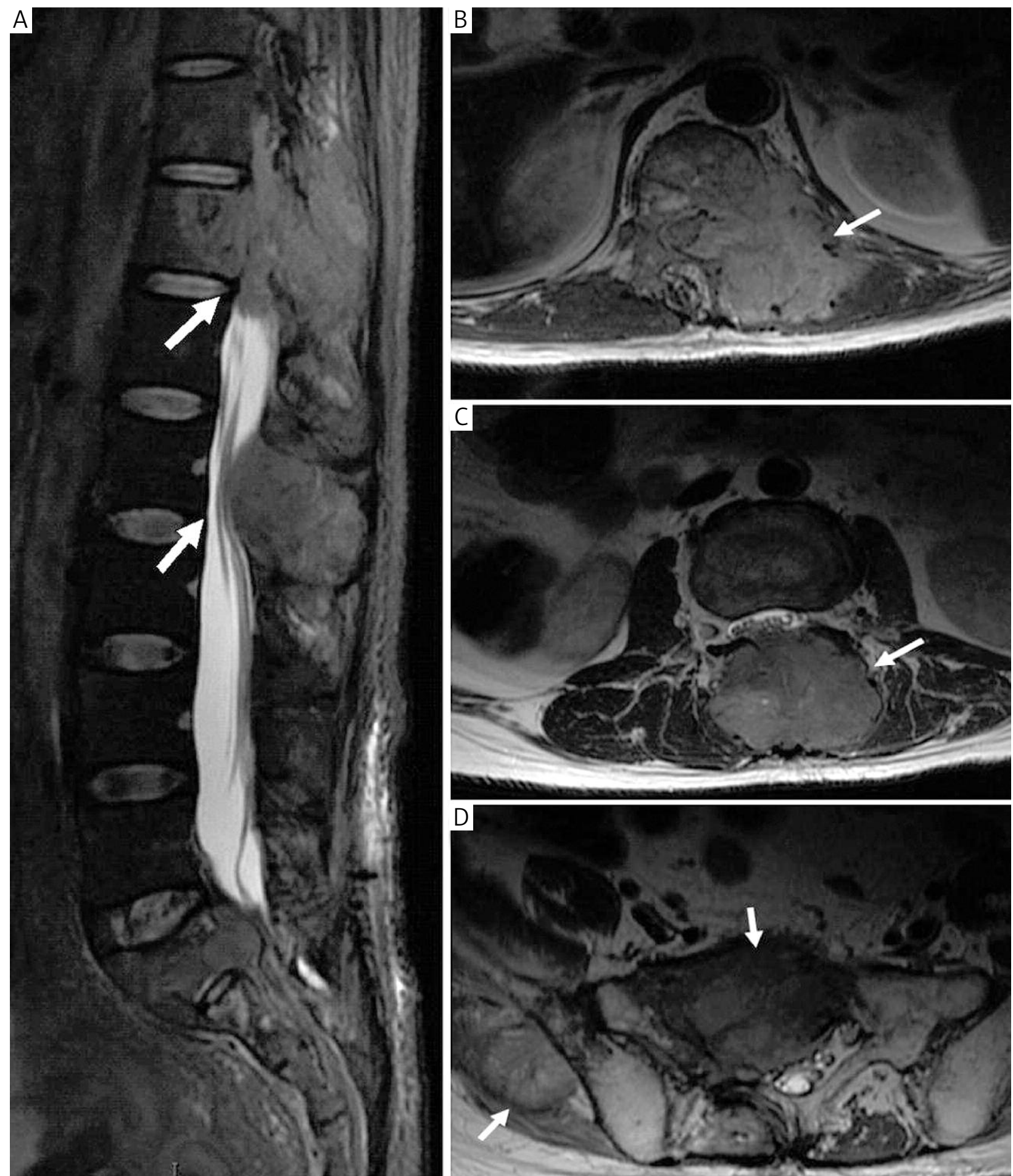

Fig. 6. A - 39-year-old man, MRI-T2WI sagittal section: equisignal soft tissue mass located at the posterior part of the vertebra in T12L2. The mass grew forwards, invading the vertebral body, and backwards, invading the paravertebral muscles; the corresponding spinal canal was replaced by soft tissue mass (indicated by arrow); B - the same patient, cross-section: peripheral mass encysted the left appendix and the adjacent vertebral body of T12; the mass invaded inwards into the spinal canal, leading to compression of the spinal cord, and was poorly defined from the paraspinal muscles (indicated by arrow); $\mathrm{C}$ - cross-section: mass centered at the spinous process of L2; it was well defined and grew forwards into the vertebral canal with a narrowed spinal cavity (indicated by arrow); D - cross-section: a relatively low signal mass was observed in the sacrum and coccyx, which was centered at the right ilium; another abnormal signal was observed inside the ilium with a poorly defined mass (indicated by arrow) 
invaded. Despite the fact that lesions in appendix sectors only accounted for $25.6 \%$, they were scattered in 51 vertebrae; chances of appendix metastasis were $52 \%$ (51 of 98) in patients with vertebral metastasis.

Some previous studies suggested that appendix metastases were an extension of lesions in the vertebral body, and appendix lesions were necessarily accompanied by vertebral body metastasis [12]. Our study discovered, however, that appendix lesions could exist separately; 12 patients were observed with appendix metastasis, but no vertebral body metastasis. In cases with both vertebral body and appendix metastases, apart from vertebral body lesions extending to the appendix sector, there were also some metastatic lesions centered at the appendix sector, extending to the vertebral body sector. Previous studies failed to address the fact that individual appendix lesions were all combined with formation of soft tissue mass, suggesting that it could be a unique feature of bone metastasis from HCC; nevertheless, it could also be a common phenomenon, only without being proposed formally.

Because of the fact that the probability of appendix metastasis is as high as $52 \%$, and some lesions exist independently of vertebral body metastasis, in HCC patients with suspected vertebral metastasis extra attention should be paid to the appendix sector when analyzing imaging data for vertebral metastasis, to avoid possible missed diagnosis. Attention should also be paid to whether lesions are outside of the target volume, or if the radiation dose is insufficient, because of missed diagnosis when the patient undergoes radiation therapy.

Another prominent characteristic of bone metastasis from HCC is that it is susceptible to formation of soft tissue mass. In our study, patients with formation of soft tissue accounted for $68.6 \%$. Previous studies also mentioned that expansive masses were easily formed at the lesion site [10, 11], which was different from bone metastasis from other types of tumor. In a handful of studies that were related to whether soft tissue was formed at the lesion site, Mizumoto reported a group of 603 patients with bone metastasis from lung, breast, gastrointestinal, prostate cancer and so forth; $18.4 \%$ were observed with soft tissue formation [13]; soft tissue mass formed in long bone metastasis from kidney cancer accounted for 30\% [14]. In our study, in a total of 232 bone metastasis patients (the most common primary tumors were lung cancer, breast cancer, gastrointestinal cancer, etc.), formation of soft tissue mass accounted for $23.3 \%$ (unpublished) - significantly lower than in the case of HCC.

Two major growth patterns of soft tissue mass have been observed: 1) the center of the mass is located at the site of bone lesion, part of which can break through the cortex and grow in the vicinity. This kind of growth is common in the vertebral body, rib and sternum; 2) another type presents as a paravertebral mass, which grows inwards to encyst and destroy bone matrix; the center of the mass seems not to be located at the metastatic bone; hence it could be named a "peripheral mass". This kind of growth is more common in the appendix sector and pelvis; the mass usually cannot be distinguished from muscles around the vertebrae, and even an MRI scan would not be able to determine if it is a muscular lesion invading the bone mass, or a cancer embolism planted on the periosteum and thereby forming a lesion which grows inwards, causing bone destruction, and outwards, forming a mass.
The formation of a soft tissue mass affects the clinical behavior of the metastatic lesion. A mass in the vertebral body grows in the direction of the vertebral canal, causing spinal canal stenosis and eventually spinal cord compression syndrome. A mass in the appendix can grow through the intervertebral foramen, or the vertebral lamina, and enter the vertebral canal, causing nerve root compression or spinal cord compression. A soft tissue mass in the pelvis can affect limb circulation due to compression of the iliac artery, causing swelling of the lower limbs and deep vein thrombosis; it can also affect the motor and sensory function of the lower limb due to compression of the affected nerve. Screening for bone metastasis is not yet a routine scan for HCC patients, and most patients will not have their diagnosis until the lesions have been formed for a certain period. Considering the characteristics of mass formation, an early screening test for bone metastasis and intervention should be conducted, which may lead to a better prognosis.

Another interesting phenomenon is that a soft tissue mass is not formed in a metastasis with pure osteoblastic change. This may be due to various interactions with cytokines. The implantation and growth of tumor cells alters the microenvironment inside the bone cavity; osteoclasts, osteoblasts and tumor cells depend on, and also restrict each other; cytokines that promote osteoblastic change may also restrict growth of tumor cells [15]. Just as the osteoblastic change that occurs after radiation therapy in bone lesions is accompanied by suppression of metastatic lesions, bone metastasis from HCC, which presents majorly as osteolytic change, increasing the activity of osteocytes by altering the microenvironment of bone lesions, is likely to suppress the growth of tumor cells.

The susceptibility of formation of soft tissue mass in bone metastasis may be relevant to the characteristics of HCC itself. Hepatocellular carcinoma is a type of tumor with rich blood supply. Vascular endothelial growth factor (VEGF), as the most important vascular growth factor, is significantly overexpressed in HCC patients, compared to patients with cirrhosis, hepatitis and so forth; for patients with and without bone metastasis, a statistical difference is also observed in the expression level of VEGF [16]. Vascular endothelial growth factor may facilitate the formation of a metastatic niche and subsequent mass.

Treatment for bone metastasis may vary depending on the formation of soft tissue mass. Palliative radiotherapy is mostly applied to patients with bone metastasis from HCC; intervention or surgery is also applied in some cases. For patients with acute spinal cord compression, surgical decompression followed by radiation therapy is superior to simple radiation therapy for preserving spinal function; however, considering other factors such as the patient's general condition, economic condition and metastasis in other organs, not all patients with spinal cord compression syndrome undergo surgical treatment; radiotherapy thus plays an important role [17]. There are various fractionation methods and radiation doses available for bone lesions, but the efficacy of different patterns may vary under particular conditions. Nakamura discovered that when bone metastasis from HCC suppresses the spinal cord, regular radiation therapy of 30 Gy in 10 fractions falls short of improving walking [18]. Zeng also discovered in his retrospective study that for bone metastasis with soft tissue mass formation, the total dose of radiotherapy should be elevated to increase the local control rate [4]. For patients with poor health condition 
and uncontrolled metastatic lesions in other organs, palliative treatment should be considered just to alleviate symptoms. For patients with fair health condition and isolated bone metastasis (without metastasis in other organs), however, location of bone metastasis and formation of soft tissue mass should also be taken into consideration; a suitable fractionation method and radiation dose should be determined in radiation therapy schedules; special irradiation technique may also be applied for better protection of normal tissue; increasing the total dose of radiotherapy may be beneficial to enhancing local control, even prolonging survival.

There were, however, also several shortcomings in our study. It was a retrospective study, and all patients were hospitalized because of symptoms that developed from metastatic bone lesions and required radiotherapy; hence, case selection was not random sampling; bone lesion destruction was relatively severe compared to asymptomatic patients. Nevertheless, by far the majority of patients with bone metastases from HCC cannot have a clear diagnosis until symptoms are observed, followed by a thorough examination. Therefore, with the expectation of bone metastasis from HCC becoming a regular screening test in future clinical practice, more early cases of bone metastasis can be discovered, and a conclusion can be made with further comparison. Also, images were analyzed based on 3-5 mm CT scans, $61.9 \%$ of which were accompanied by references to MRI data. Computed tomography showed clear trabecular and cortical structure, which was helpful in determining the extent of bone destruction and evaluating completeness of the cortex; however, when compared to MRI, it was slightly inferior in terms of revealing marginal soft tissue and fine structures of the bone cavity [19]. Not every patient had undergone an MRI scan, and some minor lesions were not optimally displayed on CT scans; omission of certain early and unobvious metastatic lesions were sometimes inevitable and thus caused errors. We expect in future clinical practice that some prospective studies will be conducted to compare the advantages and disadvantages between PET-CT, full body MRI, CT, and SPECT in diagnosing metastatic bone lesions from HCC.

In conclusion, in our study of bone metastasis from HCC, lower thoracic and lumbar vertebrae metastases were most frequently observed. Involvement of the vertebral body was most common, and distribution in various sectors showed no significant difference. Involvement of the appendix sector was also common, and could exist independently of vertebral body metastasis; hence extra attention should be paid when analyzing bone metastasis, in order to avoid missed diagnosis. Another major characteristic of bone lesions from HCC is the formation of soft tissue mass; isolated appendix sectors are more prone to forming masses, which could increase the chance of developing spinal cord compression syndrome. Listing the bone metastasis screening test as routine examination for HCC patients would be beneficial for early discovery and diagnosis, and early treatment may help improve prognosis; existence of soft tissue mass may also affect the fractionation method and total dose of radiotherapy.

The authors declare no conflict of interest.

\section{References}

1. Parkin DM, Bray F, Ferlay J, Pisani P. Global cancer statistics, 2002. CA Cancer J Clin 2005; 55: 74-108.

2. Fukutomi M, Yokota M, Chuman H, Harada H, Zaitsu Y, Funakoshi A, Wakasugi $\mathrm{H}$, Iguchi $\mathrm{H}$. Increased incidence of bone metastasis in hepatocellular carcinoma. Eur J Gastroenterol Hepatol 2001; 13: 1083-8.

3. Llovet JM, Burroughs A, Bruix J. Hepatocellular carcinoma. Lancet 2003; 362: 1907-17.

4. He J, Zeng ZC, Tang ZY, et al. Clinical features and prognostic factors in patients with bone metastasis from hepatocellular carcinoma receiving external beam radiotherapy. Cancer 2009; 115: 2710-20.

5. Chinese Liver Cancer Association. The diagnostic criteria and stages of primary hepatoma in the 4th national conference of liver cancer: problems and discussion. Chin J Gen Surg 2000; 15 : 238-9.

6. Ryder SD. Guidelines for the diagnosis and treatment of hepatocellular carcinoma (HCC) in adults. Gut 2003; 52 Suppl 3: 1-8.

7. Boriani S, Weinstein JN, Biagini R. Primary bone tumors of the spine. Terminology and surgical staging. Spine (Phila Pa 1976) 1997; 22: 1036-44.

8. Nielsen OS, Munro AJ, Tannock IF. Bone metastases: pathophysiology and management policy. J Clin Oncol 1991; 9: 509-24.

9. Okazaki N, Yoshino M, Yoshida T, Hirohashi S, Kishi K, Shimosato Y. Bone metastasis in hepatocellular carcinoma. Cancer 1985; 55: 1991-4.

10. Golimbu C, Firooznia H, Rafii M. Hepatocellular carcinoma with skeletal metastasis. Radiology 1985; 154: 617-8.

11. Kuhlman JE, Fishman EK, Leichner PK, Magid D, Order SE, Siegelman SS. Skeletal metastases from hepatoma: frequency, distribution, and radiographic features. Radiology 1986; 160: 175-8.

12. Algra PR, Heimans JJ, Valk J, Nauta JJ, Lachniet M, Van Kooten B. Do metastases in vertebrae begin in the body or the pedicles? Imaging study in 45 patients. AJR Am J Roentgenol 1992; 158: 1275-9.

13. Mizumoto M, Harada $\mathrm{H}$, Asakura $\mathrm{H}$, et al. Radiotherapy for patients with metastases to the spinal column: a review of 603 patients at Shizuoka Cancer Center Hospital. Int J Radiat Oncol Biol Phys 2011; 79: 208-13.

14. Forbes GS, McLeod RA, Hattery RR. Radiographic manifestations of bone metastases from renal carcinoma. AJR Am J Roentgenol 1977; 129: 61-6.

15. Clines GA, Guise TA. Molecular mechanisms and treatment of bone metastasis. Expert Rev Mol Med 2008; 10: e7.

16. Kaseb AO, Hanbali A, Cotant M, Hassan MM, Wollner I, Philip PA. Vascular endothelial growth factor in the management of hepatocellular carcinoma: a review of literature. Cancer 2009; 115: 4895906.

17. Patchell RA, Tibbs PA, Regine WF, Payne R, Saris S, Kryscio RJ, Mohiuddin M, Young B. Direct decompressive surgical resection in the treatment of spinal cord compression caused by metastatic cancer: a randomised trial. Lancet 2005; 366: 643-8.

18. Nakamura $\mathrm{N}$, Igaki $\mathrm{H}$, Yamashita $\mathrm{H}$, et al. A retrospective study of radiotherapy for spinal bone metastases from hepatocellular carcinoma (HCC). Jpn J Clin Oncol 2007; 37: 38-43.

19. Costelloe CM, Rohren EM, Madewell JE, et al. Imaging bone metastasis in breast cancer: techniques and recommendations for diagnosis. Lancet Oncol 2009; 10: 606-14.

\section{Address for correspondence}

\section{Yong-rui Bai}

Department of Radiation Oncology

Renji Hospital afflicated to School of Medicine

Shanghai Jiao Tong University

Shanghai, China

e-mail: Baiyongruiz@gmail.com

Submitted: 24.02 .2012

Accepted: $\quad 29.05 .2012$ 